\title{
Application of 3D-DSA simulated surgical path in intracranial aneurysm clipping surgery
}

\author{
Jun Sun ${ }^{*}$, Zhiwei Li, Chuan Lu, Xiandong Chen, Maohua Chen, Huajun Ba and Qun Lin
}

\begin{abstract}
Background: Intracranial aneurysm is a common cerebrovascular disease, which cause wide concern by neurosurgeons. The 3D-DSA images simulated surgical path may improve the success rate of operation in bridging surgery of emergency ruptured intracranial aneurysms.

Methods: Eighteen patients with intracranial ruptured aneurysms that underwent craniotomy clipping surgery from January 2015 to January 2016 were included in this study. All patients were diagnosed with ruptured aneurysm and SAH, and 3 of them had concomitant hematoma. All patients were clipped in 3 days after diagnosis. Before clipping, the relevant information and data of the 3D-DSA images simulated surgical path were observed to guide intracranial aneurysm clipping surgery.

Results: According to the information of the 3D-DSA images simulated surgical path, all aneurysms were clipped successfully. 1 patient died of vessel spasm, and the remaining patients recovered well.

Conclusions: The 3D-DSA images simulated surgical path could provide surgeons with a lot of information in bridging surgery of emergency ruptured intracranial aneurysms,and is significant to improve the success rate of operation.
\end{abstract}

Keywords: Bridging surgery, Intracranial aneurysms, 3D-DSA, Clipping surgery, Simulated surgical path

\section{Background}

Intracranial aneurysm is a common cerebrovascular disease, which cause wide concern by neurosurgeons, because of the high risk of bleeding, high morbidity and mortality [1]. Surgical clipping of intracranial aneurysm is a classic neurosurgical surgery, with an undisputed therapeutic effect. With the growing demand on surgical accuracy, minimal invasion, postoperative recovery, and the teaching quality, requirements for surgeons are also increasing. In neurosurgery department, however, these requirements have become possible to meet with the development of three-dimensional reconstruction of CT angiography (3D-CTA) and digital subtraction 3D simulation angiography (3D-DSA) images simulated surgical path. Our hospital applied the 3D-DSA images simulated surgical path on double plate DSA machine before

\footnotetext{
*Correspondence: lizhiwei10291@126.com

Department of Neurosurgery, Wenzhou Central Hospital, Wenzhou 325000, People's Republic of China

\section{Methods \\ Patient population}

A total of 18 patients were enrolled between January 1 , 2015 to January 1, 2016. There were 10 males and 8 females, aging 34-76 years, with a mean age of 58. All patients presented with sudden severe headache, with the following $\mathrm{H} \& \mathrm{H}$ grades: grade 1 in 2 cases, grade 2 in 6 cases, grade 3 in 8 cases, grade 4 in 2 cases. All of their emergency cranial CTs showed subarachnoid hemorrhage (SAH), including 3 cases accompanied with hematoma. All patients underwent CTA and DSA examination within 3 days: anterior communicating artery 
aneurysms in 8 cases, middle cerebral artery aneurysms in 5 cases, posterior communicating artery aneurysms in 3 cases, carotid artery bifurcation aneurysm in 2 cases.

\section{Surgical technique}

We performed whole neck cerebral angiography with Siemens Artis zee biplane Universal interventional radiology machine (DSA machine), in the Interventional Center. Orthophoric and lateral intracranial angiography, 3D-DSA angiography and three-dimensional reconstruction were performed in all patients under general anesthesia, with Seldinge's technique, in femoral artery approach, with 5F imaging catheter. These 18 patients aborted interventional operations due to unsuccessful interventional embolization or demand for craniotomy clipping by family members.

We simulated and designed the 3D-DSA operation path pattern with the Syngo system on DSA machine when sending the patients to the operating room. In this process, we identified blood vessels, nerves, brain, skull logo, and the location, shape, size, neck, pointing, rupture site of the aim aneurysms, and the relationship between the aneurysms and their parent arterys, adjacent blood vessels and tissues. Finalize operation plans after slight adjustment based on the classic pterion approach. Meanwhile, the patients were sent to the operating room, and got ready to craniotomy for aneurysm clipping.

\section{Results}

Craniotomy were performed in all 18 patients according to the designed approach. All patients underwent successful aneurysm clipping surgery. Some complex or unconventional aneurysms were smoothly clipped, thanks to preoperative design of the approach. After operation, subarachnoid hemorrhage was mitigated to different extent. Hematomata in three cases were also cleared. 17 cases scored 5 in the postoperative GOS, revealing a good recovery. One patient scored 1, who developed progressive brain infarctional edema and intracranial hypertension because of severe cerebral vasospasm, and died after bilateral decompressive craniectomy.

\section{Discussion}

Intracranial aneurysm is the most common cerebrovascular disease, because of the high risk of bleeding, morbidity and mortality [1]. Both interventional embolization and craniotomy clipping have been widely accepted. 3D-DSA is the "gold standard" of the diagnosis of cerebral aneurysms [2], and people are paying increasing attention to its applications. 3D-DSA images simulated surgical path guided and compared surgical procedure in the process of intervention centers bridging to Operating room. In this group of emergency patients, when embolization was infeasible or family members requested clipping surgery. Discussion is as follows (taking anterior communicating artery aneurysms for example):

\section{Approach and path design Timing}

The design process was carried out during the interval from completion of the procedure in intervention center to the operating table in the operating room. This period, lasting for $15-20 \mathrm{~min}$, was perfectly utilized to stimulate and design 3D-DSA, thus no additional time was needed.

\section{Design}

2D-DSA is not adequate to provide a comprehensive local anatomical information. In contrast, with the use of 3D-DSA, a surgeon can fully understand the spatial structure of anterior communicating artery aneurysms and parent artery before operation. This is vital to improving the safety of clipping and reducing complications $[3,4]$. 3D-DSA can provide the surgeon with full understanding of the local anatomy and simulate surgical conditions, such as relationship between the aneurysm and the parent artery, adjacent blood vessels and tissues. Therefore, we took full advantage of 3DDSA when designing. Firstly, we identified the location, shape, size, neck, pointing, rupture site of the ruptured aneurysm, and payed attention to the local anatomical relationship between the aneurysm, relevant blood vessels, nerves, brain tissue, and skull marks. Secondly, we observed the visual angle of approach after adjustment based on the classic pterion approach to determine the surgical path, the clip angle and choose the aneurysm clip. Like the cases in this study, the paths we designed before the surgery were used as the actual microscopic surgery operating position (Fig. 1). Doing so not only enhanced the confidence of surgeons, but also improved the safety and operability of the surgery.

\section{The surgical procedure}

All the operations were bridging operations, namely the doctors who performed the intervention and designed the 3D-DSA simulated path also performed the craniotomy. It was safer and more feasible, since the surgeon understood the surgical path and the aneurysm anatomy better than others.

\section{Body position and approach}

By using the simulation of both sides of pterion position, surgeons could better predict and judge the intraoperative local situation, and decide the most convenient approach to expose and clip the aneurysm. This preoperative prediction and judgment of local anatomy is significant in generating surgical strategies and preventing unexpected 

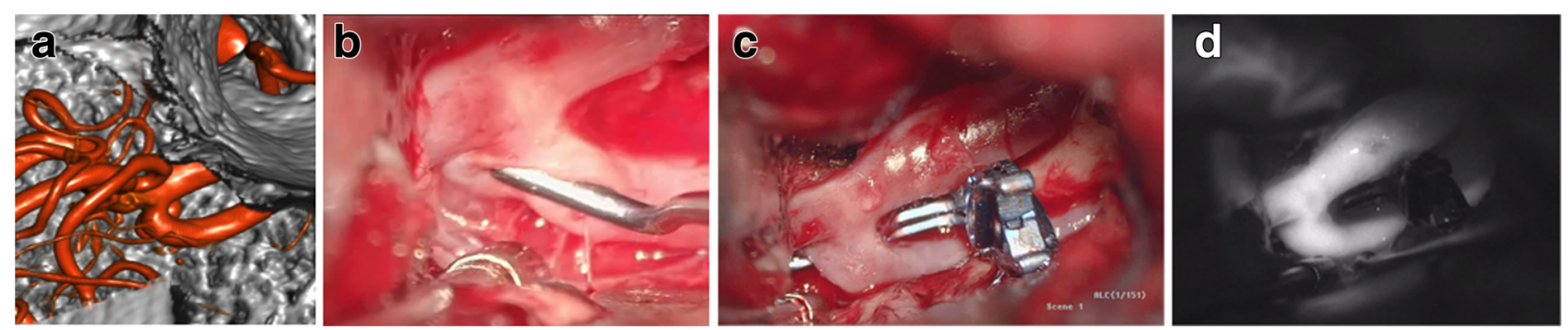

Fig. 1 The path we designed under 3D-DSA before the operation. a: 3D-DSA simulated path $\mathbf{b}$ : surrounding tissue of the parent artery c: the clip and tissue when cliping $\mathbf{d}$ : fluorenscense angiography after clipping

situations [5]. This group of patients were placed in body position and the head position based on 3D-DSA simulated approach. The surgeons sought, exposed, isolated aneurysms according to simulated approach. It could reduce unnecessary separation and tractive injury of brain tissue in the operation, and improve the operation efficiency, shorten the operation time and reduce the surgical injury.

\section{Regional anatomy}

We decided to adopt the non blood-supply dominating side pterion approach after judging the difficulty in blood-supply dominating side approach and the advantage in local exposure of the contralateral approach with the 3D-DSA. Intraoperative findings of regional anatomy was very close to 3D-DSA simulated vascular anatomy which was proved to be a good guidance [5]. The regional anatomy between the aneurysm, the parent artery and the perforating branches was very important. When we isolated the parent artery, we sought the aneurysm according to the spatial positional relationship between aneurysm and parent artery we acquired before. It greatly reduced the time we spent to separate and dissect the aneurysms, and reduced the risk of aneurysm rupture during separation. That was the reason why we were able to find and separate the aneurysm quickly, and avoid clipping other arteries by mistake (Fig. 2).

\section{Aneurysm clipping}

The aneurysm pointing must firstly be identified in 3DDSA image reconstruction, and secondly we identified the rupture position. In this way, the surgeons knew clearly the shape, size, structure, pointing and rupture position of the aneurysms before the craniotomy. Consequently, we were able to avoid injuring the structures surrounding the rupture position when exposing the aneurysm. We were able to clip the aneurysms after the neck, the parent artery, the surrounding tissue of the aneurysms were all fully exposed, thus minimize the risk of secondary ruptures (Fig. 1). In this study, one case of intraoperative bleeding occurred, but it was not during the process of separating the aneurysm.

\section{Teaching and demonstration}

Application of 3D-DSA simulated surgery path technique makes it possible for us to get better observation of stereoscopic vessel images from any angle. Also, doctors can directly understand the condition of the brain parenchyma, the parent artery and the skull base of diseased region. All of these are conducive to safer aneurysm clipping, and to intuitive teaching and display. We teach young doctors, students and interns with 3DDSA image combined with operative video after operation.

We believe that the mechanized 3D-DSA images simulated surgical path effectively makes up for the defects
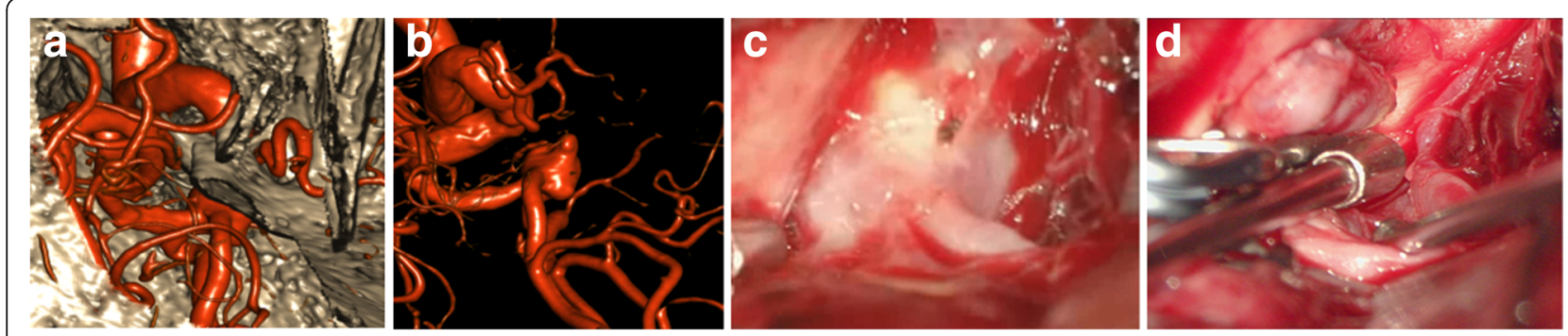

Fig. 2 3D-DSA bridging to clipping so that we can separate the aneurysm quickly. a: 3D-DSA simulated path b: 3D-DSA c: surrounding tissue of the parent artery under microscope $\mathbf{d}$ : avoid to clip contralateral A1 and perforating branches 
of 2D-DSA. It can not only guide bridging operation in emergency ruptured intracranial aneurysm, but also help in teaching. It displayed high feasibility and reliability.

\section{Researches abroad}

Researchers abroad showed the importance of 3D-DSA in the evaluation and the treatment of aneurysms [6-8]. In our study, we applied 3D-DSA to evaluate aneurysms in bridging surgery to ensure a smooth and safe process of transit.

\section{Conclusions}

The 3D-DSA simulated surgical path could provide surgeons with a lot of information in bridging surgery of emergency intracranial ruptured aneurysms, and had important significance to improve the success rate of operation.

\section{Abbreviations}

3D-DSA: 3 dimension-digital subtraction angiography; CTA: computed tomography angiography; GOS: Glasgow outcome scale; SAH: subarachnoid hemorrhage

\section{Acknowledgements}

No additional acknowledgements.

\section{Funding}

Not Applicable

\section{Availability of data and materials}

Data sharing not applicable to this article as no datasets were generated or analysed during the current study.

\section{Authors' contributions}

SJ designed and supervised the entire study and participated in drafting the manuscript. LZ and LQ searched the database. LC and CX extracted data and reported results. $\mathrm{CM}$ and $\mathrm{BH}$ reviewed the reports and provided recommendations on the discussion and conclusion. All authors read and approved the final manuscript.

\section{Ethics approval and consent to participate}

For this investigation was obtained from the Research Ethics Committee, Wenzhou central hospital. NO. 2014-04-010.

\section{Consent for publication}

We have obtained consent from all patients or their legal guardians.

\section{Competing interests}

The authors declare that they have no competing interests.

Received: 3 January 2017 Accepted: 30 November 2017

Published online: 14 December 2017

\section{References}

1. Hop JW, Rinkel GJ, Algra A, Van Gijn J. Case-fatality rates and functional outcome after subarachnoid hemorrhage: a systematic review. Stroke. 1997; 28:660-4.

2. van Rooij WJ, Sprengers ME, de Gast AN, Peluso JP, Sluzewski M. 3D rotational angiography: the new gold standard in the detection of additional intracranial aneurysms. AJNR Am J Neuroradiol. 2008;29:976-9.

3. Lauriola W, Nardella M, Strizzi V, Cali A, D'Angelo V, Florio F. 3D angiography in the evaluation of intracranial aneurysms before and after treatment. Initial experience. Radiol Med. 2005;109:98-107.

4. Unger B, Link J, Trenkler J, Bohm-Jurkovic H. Digital 3D rotational angiography for the preoperative and preinterventional clarification of cerebral arterial aneurysms. Rofo. 1999;170:482-91.
5. Jun LI, Zhang G, Chen G, Qin SZ, Lian-Ting MA, Gong J, et al. Clipping of anterior communicating artery aneurysms by microsurgery through pterional approach contralateral to supply of dominant blood (report of 12 cases). Chin J Clin Neuro. 2011;16:327-8.

6. Wong SC, Nawawi O, Ramli N, Abd Kadir KA. Benefits of 3D rotational DSA compared with 2D DSA in the evaluation of intracranial aneurysm. Acad Radiol. 2012;19:701-7.

7. Missler U, Hundt C, Wiesmann M, Mayer T, Bruckmann H. Three-dimensional reconstructed rotational digital subtraction angiography in planning treatment of intracranial aneurysms. Eur Radiol. 2000;10:564-8.

8. Gauvrit JY, Leclerc X, Vermandel M, Lubicz B, Despretz D, Lejeune JP, et al. $3 D$ rotational angiography: use of propeller rotation for the evaluation of intracranial aneurysms. AJNR Am J Neuroradiol. 2005;26:163-5.
Submit your next manuscript to BioMed Central and we will help you at every step:

- We accept pre-submission inquiries

- Our selector tool helps you to find the most relevant journal

- We provide round the clock customer support

- Convenient online submission

- Thorough peer review

- Inclusion in PubMed and all major indexing services

- Maximum visibility for your research

Submit your manuscript at www.biomedcentral.com/submit
) Biomed Central 